\title{
Psychophysiological Intervention to Improve Preparedness in Military Special Operations Forces
}

\author{
José Francisco Tornero-Aguilera; Jailton Gregório Pelarigo; Vicente Javier Clemente-Suárez
}

\begin{abstract}
BACKGROUND: Actual studies in military training support the use of new methodological approaches such as high intensity interval training and inverse periodization training rather than conventional approaches. However, the application and analysis of success of these new methodologies are as yet unknown.
\end{abstract}

METHODS: The military adapted the civil reverse periodization training system, composed of 6 wk and five sessions per week. For the first 3 wk soldiers performed two sessions with the objective of increasing the maximal strength of the upper and lower body muscles, and three sessions of short high-intensity interval training (HIIT). In the following 3 wk they combined two endurance HIIT and two resistance HIIT sessions with military equipment while conducting a military task.

RESULTS: After the training a significant body mass index decrease was found, along with an increase in lower limb muscular strength, aerobic and anaerobic performance, resilience, stress tolerance, and psychological flexibility. Regarding the ratio of acceptance there was an increased ratio compared to previous years of $7 \%$. In addition, married soldiers with children presented greater resilience, stress tolerance, psychological flexibility, and a higher ratio of success.

DISCUSSION: Accepted soldiers presented greater psychological status and stress tolerance, highlighting the importance of the work of the unit psychologists to reinforce and monitor the psychometric profile of the soldiers as well as their intrinsic characteristics of personality and emotionality. The greater baseline physical condition and higher performance in all physical tests proves how reverse periodization training models are a great stimuli and training approach in soldiers.

KEYWORDS: stress, psychological flexibility, performance, periodization, aerobic performance.

Tornero-Aguilera JF, Gregório Pelarigo J, Clemente-Suarez VJ. Psychophysiological intervention to improve preparedness in military Special Operations forces. Aerosp Med Hum Perform. 2019; 90(11):1-6.

ongitudinal and descriptive studies show an increased tendency of obesity, ${ }^{23}$ psychological disorders, ${ }^{22}$ and a general decrease in physical capacity ${ }^{18}$ in young military personnel. This phenomenon is a big challenge for military training and recruitment of capable soldiers, since modern military operations are highly physically demanding. ${ }^{12}$ Besides military skills and physical performance, a successful military operation, especially in actual theaters of operations, also requires a good psychological status, self-confidence, and readiness. ${ }^{11,29}$ These actual psychophysiological demands for warfighters identified them as "tactical athletes," 21 eliciting in them in military training, essential military knowledge, military skills, and physical and psychological capacities. ${ }^{25}$

These tactical athletes must face physiological demands such as increases in sympathetic modulation, blood lactate concentrations, exceeding the anaerobic threshold similarly to elite athletes, ${ }^{12}$ large muscular and cardiovascular responses, and a down regulation of cortical arousal. ${ }^{5}$ However, regarding the stressful nature of military combat, authors also describe psychological alterations in perception and memory dysregulation as an acute effect of stress. ${ }^{16,17}$ In this line, new technological innovations have not reduced the physical demands of soldiers; however, these may attenuate psychological stress, such as the use of night vision systems in underground operations. $^{29}$ These psychophysiological demands in these stressful

From the Research Center in Applied Combat (CESCA), Toledo, Spain; the Universidad Europea de Madrid, Madrid, Spain; the Metropolitan College of Grande Fortaleza, Fametro, Fortaleza, Ceará, Brazil; and the University Catholic Center of Quixadá Unicatólica, Quixadá, Ceará, Brazil.

This manuscript was received for review in March 2019. It was accepted for publication in July 2019.

Address correspondence to: Vicente Javier Clemente-Suárez, Faculty of Sport Sciences. Tajo street, s/n, 28670 Villaviciosa de Odón, Madrid, España; vctxente@yahoo.es.

Reprint \& Copyright $\odot$ by the Aerospace Medical Association, Alexandria, VA.

DOI: https://doi.org/10.3357/AMHP.5385.2019 
environments have been widely reported either in close quarter, symmetrical and asymmetrical combat, or tactical parachute jumps, ${ }^{30-32}$ concluding there is a necessity to include specific training models adapted to the military service which consider the specific psychophysiological needs of the tactical athlete.

Actual military training approaches have been focused either on the increase of physical fitness ${ }^{19}$ or on the decrease of soldiers' injuries and risks factors. ${ }^{3}$ However, these programs are performed in a low stress environment, a situation opposite to real combat. Thus, successful operations not only require military skills, proper tactics knowledge, and advanced armor, instruments, and systems, but good psychophysiological performance too. Therefore, identifying the most appropriate physical and psychological approach is essential for the training of new recruits, which is a key factor for developing future soldiers with improved mission survival and success ratio, especially in high demand units like special operations. For this reason, we conducted the present study with the aims of: 1) analyzing psychophysiological modifications after an experimental operative training previous to a special operations selection course; and 2) analyzing the ratio of candidates approved after performing the experimental training in a special operations selection course. We hypothesized that the training program would improve the psychophysiological profiles of recruits, increasing the ratio of acceptance in the special operations selection course compared with previous years.

\section{METHODS}

\section{Subjects}

A sample of 61 volunteer participants were recruited. All of them were aspirants for a special operations selection course of the Spanish Army (24.9 \pm 4.7 mean age; $177.8 \pm 6.0 \mathrm{~cm} ; 77.4 \pm$ $9.6 \mathrm{~kg} ; 24.6 \pm 3.2$ body mass index; $1.4 \pm 4.4 \mathrm{yr}$ of military experience; $0.2 \pm 0.5 \mathrm{yr}$ in their unit; $0.2 \pm 1.1 \mathrm{yr}$ on international missions). Prior to starting the research, the experimental procedures were explained to all the participants, who gave their voluntary written informed consent in accordance with the Declaration of Helsinki. The procedures conducted in the present research were designed and approved by the Headquarters of the unit (e thical committee of clinical research with medicines of the Hospital central de la defensa; Code: 68/18).

\section{Equipment}

The following dependent variables were analyzed before and after completing the training. Body mass was measured using a Seca scale (model 714; Seca, Hamburg, Germany) with a precision of $100 \mathrm{~g}$ (range $0.1-130 \mathrm{~kg}$ ) located on a flat and smooth surface and calibrated at zero. Subjects were barefoot with minimal clothes. Once located in the center of the platform, they remained still without their body being in contact with surrounding objects, with the mass evenly distributed on both feet facing forward.

Height was measured with a height rod incorporated in the Seca scale with a precision of $0.1 \mathrm{~mm}$ (range $60-200 \mathrm{~cm}$ ). The subject stood up, barefoot, with the head oriented in the Frankfort plane that joins the inner edge of the eye socket and the upper one of the external auditory meatus, arms on both sides of the trunk, extended and with palms touching the external face of the thighs, heels together touching the lower end of the vertical surface with the inner edge of the feet at 45 to $60^{\circ}$, with the occipital area, scapular, buttocks, and the posterior face of the knees and calves touching the vertical surface of the anthropometer.

Body mass index (BMI $=$ mass in $\mathrm{kg} /$ height $^{2}$ in $\left.\mathrm{m}\right)$ was calculated according to the World Health Organization.

Lower limb strength manifestation was measured through a maximal horizontal jump test. Subjects performed a standardized warm-up consisting of 10 min of running (light aerobic intensity). Then, participants performed two maximal horizontal jumps as in a previous report. ${ }^{15}$ Both jumps were performed with the hands on the waist to avoid inertial arm movement and the best attempt was used for the statistical analysis.

Running performance was measured through two tests. First a 50-m run and a 2000-m run at maximal effort, with the last being associated with the maximal aerobic speed measured in the laboratory test. ${ }^{15}$ After the horizontal jump test, participants were instructed to run $50 \mathrm{~m}$ at maximal speed, rest $10 \mathrm{~min}$, and then start the 2000-m run at maximal speed on a regulation competition track field (temperature $16.5 \pm 1.2^{\circ} \mathrm{C} ; 62.2 \pm 1.2 \%$ humidity). The time to complete both tests and heart rate (HR) were recorded. HR was measured using a Polar V800 HR monitor (Polar Electro, Kempele, Finland).

Life Engagement Test (LET). This scale has 6 items and it was designed to measure the purpose of people in life. In this line, this scale analyzes the degree of involvement of the person in activities that are important within their daily surroundings. Item Example: "For me, all the things I do deserve the punishment." Answers on a Likert scale of 1 to 5 where $1=$ Strongly disagree and $5=$ Strongly agree. ${ }^{26}$

The Coping Flexibility Scale (CFS). This scale was designed to measure the flexibility in coping with different situations. It refers to the presence of adaptive coping strategies that are associated with better psychological health. This test has 10 items and is answered on a Likert scale of 1 to 4 , with $1=$ very applicable to $4=$ not applicable. $^{24}$

Perceived Stress Scale (PSS). This scale assesses the level of perceived stress in a 1-mo period. It is composed of 14 items that are answered with a 5 -point Likert scale, where $0=$ never and $4=$ very often. High scores are related with a higher perception of stress. $^{24}$

Attitudes to Ageing Questionnaire (AAQ). This scale assesses the subjective perception of ageing focusing primarily on three different aspects of ageing. The first subscale focuses on psychosocial losses relevant to older adults in which the perceived negative experiences of ageing are collected together in a single composite scale. The second subscale (physical change) has a 
more mixed physical functioning focus with items related primarily to health, exercise, and the experience of ageing itself. The third subscale (psychological growth) has an explicitly positive focus and could be summarized as 'wisdom' or 'growth' as it recognizes a lifespan development perspective on ageing as viewed by the individual. ${ }^{4}$

\section{Procedures}

A military adaptation of civil reverse periodization training was applied as in previous research. ${ }^{9,13,14}$ This new training model is based on specific high-intensity and low-volume training, which are some of the training methodologies recently proposed as basic for military training in actual theaters of operations. ${ }^{14}$

Training was composed of $6 \mathrm{wk}$ with five sessions per week having a duration between 30 min during the first week and $50 \mathrm{~min}$ the following weeks. During the first $3 \mathrm{wk}$ soldiers performed two sessions at a gym with the objective of increasing maximal strength of the upper and lower body muscles (load between $70-85 \%$ of the maximum strength) and three sessions of short high-intensity interval training (HIIT).$^{30}$ In the following $3 \mathrm{wk}$ they combined two short HIIT sessions and two resistance HIIT with military equipment while conducting a military task.

\section{Statistical Analysis}

To analyze the data, we used the SPSS statistical package (version 22.0; SPSS, Inc., Chicago, IL, USA). Means and SD were calculated using traditional statistical techniques. Normality and homoscedasticity assumptions were checked with a Kolmogorov-Smirnov test. To analyze differences between preand post-training samples, a $t$-test for dependent samples was administered and to analyze differences between accepted and non-accepted participants in the operation selection course, a $t$-test for independent samples was used. The effect size (ES) was tested by Cohen's $d$ [ES = (Posttest mean - Pretest mean)/ Pretest SD]. The level of significance for all the comparisons was set at alpha $<0.05$.

\section{RESULTS}

The results are reported as mean $\pm \mathrm{SD}$. Significant body mass and BMI decrease were found. Regarding physical performance, a significant increase in the 50-m, 2-km tests and lower limb strength manifestation was measured, as well as in rating of perceived exertion (RPE) values. In relation to the psychometric tests, a significant increase was found in the LET test, and the PSS the AAQ tests presented a significant decrease. Finally, no significant changes were found for the CFS (Table I).

Soldiers with higher age and experience, less body mass, and less BMI presented a higher ratio of success. In addition, higher scores on the LET and CFS psychometric tests as well as being married and with children, predicted a greater ratio of acceptance. Finally, $77 \%$ of the candidates were accepted and $23 \%$ were rejected; this ratio of accepted candidates was higher than previous years, where this ratio was between 65-70\% (Table II).

\section{DISCUSSION}

The aim of the present research was to analyze the psychophysiological modifications after an experimental operative training previous to a special operations selection course and the ratio of candidates approved after performing the experimental training. The initial hypothesis was confirmed since the psychophysiological profile of the recruits improved and the ratio of soldiers' acceptance in the operation selection course was higher than in previous years.

Previous researches conducted in urban combat, ${ }^{11,12}$ symmetrical and asymmetrical combat, ${ }^{14,31}$ close quarter combat, ${ }^{10}$ and tactical parachute jumps ${ }^{5,8,9}$ showed a high activation of the anaerobic metabolism and a high demand on aerobic capacity, highlighting the importance of training both metabolic pathways. In this line, the HIIT used in the present training program and proposed as an effective tool to improve the anaerobic metabolic pathway ${ }^{6}$ resulted in a performance improvement,

Table I. Mean \pm SD Values for the Psychophysiological Parameters Analyzed Before and After Training.

\begin{tabular}{|c|c|c|c|c|c|c|c|c|}
\hline & \multirow[b]{2}{*}{ PRE } & \multirow[b]{2}{*}{ POST } & \multirow[b]{2}{*}{ COHEN'S d } & \multirow[b]{2}{*}{$\%$ OF CHANGE } & \multirow[b]{2}{*}{$\Delta$} & \multirow[b]{2}{*}{$P$} & \multicolumn{2}{|c|}{$\begin{array}{c}95 \% \mathrm{Cl} \text { OF } \\
\text { THE DIFFERENCES }\end{array}$} \\
\hline & & & & & & & LOWER & UPPER \\
\hline Weight (kg) & $77.7 \pm 9.8$ & $76.0 \pm 9.6$ & 0.17 & $-1.76 \pm-0.16$ & 9.840 & 0.000 & 1.390 & 2.341 \\
\hline $\mathrm{BMI}\left(\mathrm{kg} \cdot \mathrm{m}^{-2}\right)$ & $25.6 \pm 1.18$ & $24.0 \pm 3.12$ & 0.17 & $-0.55 \pm-0.03$ & 9.430 & 0.009 & 0.438 & 0.671 \\
\hline Horizontal jump (cm) & $214.7 \pm 25.4$ & $219.8 \pm 24.9$ & -0.20 & $5.11 \pm 0.98$ & -2.742 & 0.008 & -8.894 & -1.389 \\
\hline $50 \mathrm{~m}$ (seconds) & $7.7 \pm 0.6$ & $7.4 \pm 0.5$ & 0.53 & $-0.34 \pm 0.05$ & 7.764 & 0.000 & 0.258 & 0.438 \\
\hline 50 m HR (bpm) & $181.8 \pm 15.2$ & $192.6 \pm 10.1$ & -0.3 & $11.8 \pm 7.06$ & -2.954 & 0.000 & -15.293 & -5.346 \\
\hline RPE $50 \mathrm{~m}$ & $11.2 \pm 2.4$ & $14.6 \pm 2.5$ & -1.36 & $3.32 \pm 0.10$ & -10.308 & 0.000 & -3.973 & -2.689 \\
\hline 2 km (seconds) & $540 \pm 4.8$ & $480 \pm 4.8$ & 0.53 & $-0.45 \pm 0.03$ & 7.654 & 0.000 & 0.343 & 0.578 \\
\hline 2 km HR (bpm) & $161.8 \pm 19.2$ & $172.6 \pm 11.1$ & -0.5 & $10.8 \pm 8.06$ & -3.954 & 0.000 & -16.293 & -5.346 \\
\hline RPE $2 \mathrm{~km}$ & $15.2 \pm 1.7$ & $15.8 \pm 1.9$ & -0.3 & $0.6 \pm 0.10$ & -2.087 & 0.041 & -1.245 & -0.026 \\
\hline LET & $17.4 \pm 1.3$ & $26.8 \pm 2.6$ & -7.46 & $9.4 \pm-1.4$ & -25.013 & 0.000 & -10.233 & -8.717 \\
\hline CFS & $18.7 \pm 2.9$ & $18.1 \pm 4.4$ & 0.21 & $-0.6 \pm 1.5$ & 0.942 & 0.350 & -0.699 & 1.954 \\
\hline PSS & $30.2 \pm 3.9$ & $18.8 \pm 7.1$ & 2.87 & $-11.4 \pm 3.1$ & 10.717 & 0.000 & 9.280 & 13.539 \\
\hline AAQ & $6.4 \pm 4.1$ & $10.9 \pm 3.2$ & -1.10 & $-4.5 \pm 0.9$ & 7.045 & 0.000 & 3.169 & 5.683 \\
\hline
\end{tabular}

BMI: body mass index; RPE: rate of perceived exertion; HR: heart rate; LET: Life Engagement Test; CFS: Coping Flexibility Scale; PSS: Perceived Stress Scale; AAQ: Attitudes to Ageing Questionnaire. 
Table II. Mean \pm SD Values for the Psychophysiological Variables Analyzed in Relation to Acceptance After the Special Operation Selection Course.

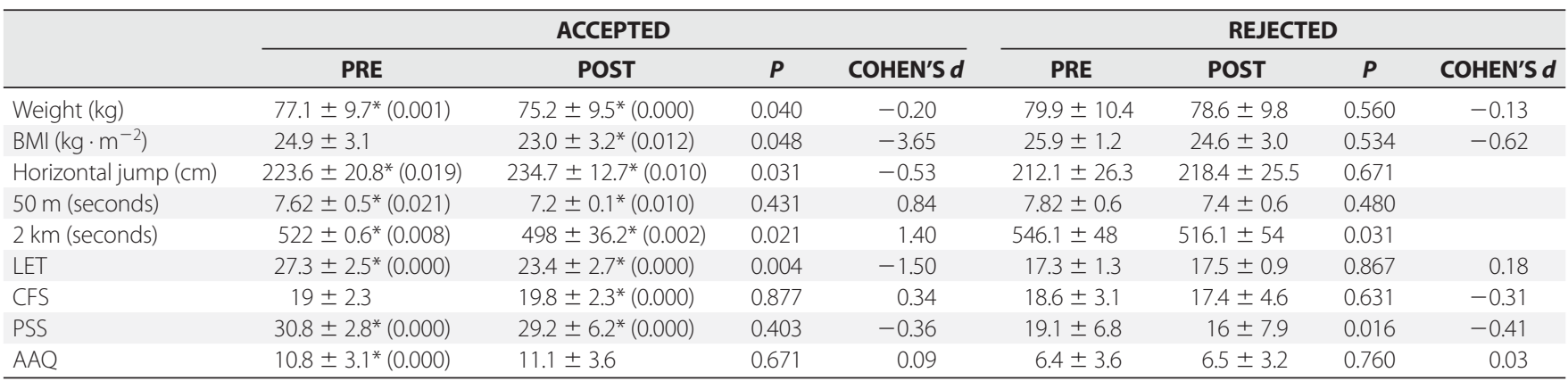

BMI: body mass index; RPE: rate of perceived exertion; HR: heart rate; LET: Life Engagement Test; CFS: Copy Flexibility Scale; PSS: Perceived Stress Scale; AAQ: Attitudes to Ageing Questionnaire

* $P<0.05$ between accepted and rejected.

showing a significant decrease in the $50-\mathrm{m}$ test time. In addition, HIIT has also been proven to be effective for increasing the maximal oxygen uptake and aerobic endurance, ${ }^{14}$ which is in line with the significant performance increase measured in the 2-km test. When analyzing differences between accepted and rejected soldiers, the first ones presented higher baseline levels of aerobic and anaerobic capacity as well as a better adaptive response, presenting higher post results in both the $50-\mathrm{m}$ and 2-km tests, which highlights the importance of optimal physical fitness in order to succeed in the special operations selection course.

The cardiovascular response in both the $50-\mathrm{m}$ and $2-\mathrm{km}$ tests supported the training program efficiency, since the significant HR increases showed higher cardiovascular adaptation and, therefore, greater ability to perform both tests at greater intensity, consequent with the time reduction found in the $50-\mathrm{m}$ and $2-\mathrm{km}$ tests. In addition, the training program could elicit and reach the cardiovascular requirements of actual combat scenarios, ${ }^{30-32}$ a fact essential for soldiers' operability and performance. The RPE modification found after training also sustained the previous affirmations, since the significant increases were in line with the ability to perform the test with higher intensity and effort, as well as to endure and tolerate higher effort, a vital fact in the high demand and stressful field of special operations activities. However, a misinterpretation of the RPE has been reported in this population in different combat situations. ${ }^{2,29}$ This fact was related to the increased sympathetic activation and negative effect in cortical structures such as the hippocampus and brain areas related to information processing. ${ }^{16,17}$ Therefore, caution should be taken when interpreting RPE results in soldiers. Future research should try to address a new subjective effort scale for this population.

Muscle strength capabilities are basic for soldiers, since they must conduct their missions with the extra weight of equipment and actions conducted in the battlefield require high manifestations of strength and endurance, independently of the previous level of training and experience, especially on the lower limbs. ${ }^{16}$ In this line, previous authors reported that experienced soldiers presented higher levels of muscular strength than new soldiers, ${ }^{29,31,32}$ a fact consequent with our data since accepted soldiers had the most experience and presented higher significant pre and post lower limb muscular strength than the rejected ones. Regarding the possible training interferences reported by authors due to the difference between the mechanisms of adaptation induced by resistance and endurancebased training, since each exercise activates or represses specific subsets of genes and cellular signaling pathways, ${ }^{20}$ no negative interferences were found when resistance exercise and HIIT were administered together. ${ }^{33}$ This result was in agreement with our data regarding the parallel increase in lower muscular strength and 50-m and 2-km performance. Regarding body composition, both accepted and rejected soldiers presented with an overweight BMI, consequent with the tendency of obesity among young military personnel in recent years. ${ }^{23}$ However, both groups showed a significant BMI decrease, most acutely in accepted soldiers. These results are in accordance with similar interventions in civil populations. ${ }^{27}$ In addition, BMI is correlated with physical performance, ${ }^{28}$ which would explain the higher ratio of success and performance of accepted soldiers in all physical tests.

According to the psychometric analysis, improvements in resilience, stress tolerance, and psychological flexibility were found, all of them basic constructs for soldiers who must face highly stressful and demanding situations. Specifically, accepted soldiers presented higher significant increases on the psychometric tests-LET, CFS, and AAQ-showing an ideal psychological status, behaviors, and stress tolerance, which lead them to success in the special operations selection course. In relation to the PSS scale, there was a significant decrease after the training program, probably due to the complexity of the training and the added stress of the special operations selection course, as it tested physical and psychological capacities, adding a considerable amount of stress. In this line, authors showed that working under stress could improve working memory and strategy selection, justifying the success of accepted soldiers ${ }^{1}$ and the reason for the higher significant PSS pre and post values when compared to rejected soldiers. Finally, our data led us to improve the knowledge of the psychophysiological variables that predispose success on the special operations selection course since the ratio of candidates accepted increased compared with previous years, making the training program a success since $77 \%$ of the candidate were accepted; in previous years 
this ratio was between $65-70 \%$. This result allows us to improve knowledge regarding the effectiveness of military training programs that would improve soldiers' performance.

In conclusion, this research showed how a specific training program for a special operations selection course increased muscular strength, aerobic and anaerobic performance, resilience, stress tolerance, and psychological flexibility, and the ratio of candidates accepted increased compared with previous years. Finally, accepted soldiers presented better psychological status and stress tolerance, highlighting the importance of the work of psychologists in the units, which is to reinforce and monitor the psychometric profiles of the soldiers as well as their intrinsic characteristics of personality and emotionality. In addition, accepted soldiers presented better baseline physical condition and higher performance in all physical tests, proving how reverse periodization training models are effective not only in a civil population (athletes), but also in a military population. Thus the direct application of this knowledge and new training approach could be used by other tactical athletes such as firefighters and police, as well as private security corps, and other units and brigades in where there is a large physical demand in their preparation and considerable stress as part of their duty. However, the lack of hormone analysis and direct evaluation of maximal oxygen uptake were limitations of the present study, which was subjected to a financial and technological lack, something future research might seek to address.

\section{ACKNOWLEDGMENTS}

We want to acknowledge the contribution of the Escuadrón de Apoyo al Desembarco Aereo (EADA) of the Spanish Air Force. declare.

Financial Disclosure Statement: The authors have no competing interests to

Authors and affiliations: Jose Francisco Tornero-Aguilera, M.Sc., Ph.D., and Vincente Javier Clemente-Suarez, Ph.D., Ph.D., European University of Madrid, Madrid, Spain; and Jailton Gregorio Pelarigo, M.Sc., Ph.D., University Catholic Center of Quixada, Quixada, Ceara, Brazil.

\section{REFERENCES}

1. Beilock SL, DeCaro MS. From poor performance to success under stress: working memory, strategy selection, and mathematical problem solving under pressure. J Exp Psychol Learn Mem Cogn. 2007; 33(6):983-998.

2. Buchheit M, Laursen PB. High-intensity interval training, solutions to the programming puzzle. Sports Med. 2013; 43(5):313-338.

3. Bullock SH, Jones BH, Gilchrist J, Marshall SW. Prevention of physical training-related injuries: recommendations for the military and other active populations based on expedited systematic reviews. Am J Prev Med. 2010; 38(1):S156-S181.

4. Chachamovich E, Fleck M, Laidlaw K, Power M. Impact of major depression and subsyndromal symptoms on quality of life and attitudes toward aging in an international sample of older adults. Gerontologist. 2008; 48(5):593-602.

5. Clemente-Suárez VJ. Periodized training achieves better autonomic modulation and aerobic performance than non-periodized training. J Sports Med Phys Fitness. 2018; 58(11):1559-1564.

6. Clemente-Suárez VJ, Dalamitros AA, Nikolaidis PT. The effect of a short-term training period on physiological parameters and running performance: intensity distribution versus constant-intensity exercise. J Sports Med Phys Fitness. 2018; 58(1-2):1-7.

7. Clemente-Suárez VJ, Delgado-Moreno R, González B, Ortega J, RamosCampo DJ. Amateur endurance triathletes' performance is improved independently of volume or intensity based training. Physiol Behav. 2019; 205:2-8.

8. Clemente-Suárez VJ, Delgado-Moreno R, González-Gómez B, Robles-Pérez JJ. Respuesta psicofisiológica en un salto táctico paracaidista HAHO: caso de Estudio. Sanid Mil. 2015; 71(3):179182.

9. Clemente-Suárez VJ, Fernandes RJ, Arroyo-Toledo JJ, Figueiredo P, González-Ravé JM, Vilas-Boas JP. Autonomic adaptation after traditional and reverse swimming training periodizations. Acta Physiol Hung. 2015; 102(1):105-113.

10. Clemente-Suarez VJ, Palomera PR, Robles-Pérez JJ. Psycho-physiological response to acute-high-stress combat situations in professional soldiers Stress Health. 2018; 34(2):247-252.

11. Clemente-Suárez VJ, Robles-Pérez JJ. Organic response in a combat simulation. Sanid Mil. 2012; 68(2):97-100.

12. Clemente-Suárez VJ, Robles-Pérez JJ. Analysis of physiological markers, cortical activation and strength manifestations in a simulated combat. Arch Med Deporte. 2012; 2012(149):594-600.

13. Clemente-Suárez VJ, Robles-Pérez JJ. Psycho-physiological response of soldiers in urban combat. Annee Psychol. 2013; 29(2):598-603.

14. Clemente-Suárez VJ, Robles-Pérez JJ. Mechanical, physical, and physiological analysis of symmetrical and asymmetrical combat. J Strength Cond Res. 2013; 27(9):2420-2426.

15. Coutts AJ, Slattery KM, Wallace LK. Practical tests for monitoring performance, fatigue and recovery in triathletes. J Sci Med Sport. 2007; 10(6):372-381.

16. Delgado-Moreno R, Robles-Pérez JJ, Aznar S, Clemente-Suarez VJ. Inalambric biofeedback devices to analyze strength manifestation in military population. J Med Syst. 2018; 42(4):60.

17. Delgado-Moreno R, Robles-Pérez JJ, Clemente-Suárez VJ. Combat stress decreases memory of warfighters in action. J Med Syst. 2017; 41(8): 124 .

18. Friedl KE, Knapik JJ, Häkkinen K, Baumgartner N, Groeller H, et al. Perspectives on aerobic and strength influences on military physical readiness: report of an international military physiology roundtable. J Strength Cond Res. 2015; 29(Suppl. 11):S10-S23.

19. Harman EA, Gutekunst DJ, Frykman PN, Nindl BC, Alemany JA, et al. Effects of two different eight-week training programs on military physical performance. J Strength Cond Res. 2008; 22(2):524-534.

20. Hawley JA. Molecular responses to strength and endurance training: are they incompatible? Appl Physiol Nutr Metab. 2009; 34(3):355361.

21. Heinrich KM, Spencer V, Fehl N, Carlos Poston WS. Mission essential fitness: comparison of functional circuit training to traditional Army physical training for active duty military. Mil Med. 2012; 177(10):11251130.

22. Hoge CW, Auchterlonie JL, Milliken CS. Mental health problems, use of mental health services, and attrition from military service after returning from deployment to Iraq or Afghanistan. JAMA. 2006; 295(9): 1023-1032.

23. Hsu LL, Nevin RL, Tobler SK, Rubertone MV. Trends in overweight and obesity among 18-year-old applicants to the United States military, 19932006. J Adolesc Health. 2007; 41(6):610-612.

24. Kato T. Development of the Coping Flexibility Scale: Evidence for the coping flexibility hypothesis. J Couns Psychol. 2012; 59(2):262-273.

25. Santtila M, Pihlainen K, Viskari J, Kyröläinen H. Optimal physical training during military basic training period. J Strength Cond Res. 2015; 29(Suppl. 11):S154-S157.

26. Scheier MF, Wrosch C, Baum A, Cohen S, Martire LM, et al. The life engagement test: assessing purpose in life. J Behav Med. 2006; 29(3):291298.

27. Schoenfeld B, Dawes J. High-intensity interval training: applications for general fitness training. Strength Condit J. 2009; 31(6):44-46. 
28. Sedeaud A, Marc A, Marck A, Dor F, Schipman J, et al. BMI, a performance parameter for speed improvement. PLoS One. 2014; 9(2):e90183.

29. Tornero-Aguilera JF, Clemente-Suarez VJ. Effect of experience, equipment and fire actions in psychophysiological response and memory of soldiers in actual underground operations. Int J Psychophysiol. 2018; 128:40-46.

30. Tornero-Aguilera JG, Clemente-Suárez VJ. Resisted and endurance high intensity interval training for combat preparedness. Aerosp Med Hum Perform. 2019; 90(1):32-36.
31. Tornero-Aguilera JF, Robles-Pérez JJ, Clemente-Suárez VJ. Effect of combat stress in the psychophysiological response of elite and non-elite soldiers. J Med Syst. 2017; 41(6):100.

32. Tornero-Aguilera JF, Robles-Pérez JJ, Clemente-Suárez VJ. Use of psychophysiological portable devices to analyse stress response in different experienced soldiers. J Med Syst. 2018; 42(4):75.

33. Wong PL, Chaouachi A, Chamari K, Dellal A, Wisloff U. Effect of preseason concurrent muscular strength and high-intensity interval training in professional soccer players. J Strength Cond Res. 2010; 24(3):653-660. 


\section{Author Query sheet-AMHP5385}

Q1 : Author: This manuscript has been lightly edited for clarity and proper English. Please read carefully to ensure it still says what you wanted.

Q2 : Author: Original Refs. 1, 6, 9, 10, 13, 17, \& 24 were deleted per our email conversation. In addition, original Refs. 7, 8, 11, 12, and 14-16, 18-23, and 37-39 were re-organized to be in alphabetical order. The references were then renumbered. Please check that the citations were correctly transposed in the text.

Q3 : Author: Does this sentence say what you meant it to say? It originally said "In relation to the psychometric tests, a significant increase was found in LET, PSS and AAQ test presented a significant decrease." 